\title{
Electron emission in asymmetric collisions with fast, heavy projectiles via continuum charge transfer
}

\author{
D H Jakubassa-Amundsen ${ }^{\dagger}$ \\ Physik-Department, Technische Universität München, Garching, West Germany
}

Received 13 October 1982, in final form 24 January 1983

\begin{abstract}
The ejection of electrons from light target atoms by bare heavy projectiles is described by means of electron capture to the continuum. Calculations of the energy distribution of the electrons are performed within the semiclassical impulse approximation and compared with other theories. As special cases, collisions of $\mathrm{Kr}$ with $\mathrm{H}$ and $\mathrm{Ar}$ with $\mathrm{He}$ are considered. Experimental data of the electron spectrum in the forward peak region in $(\mathrm{Ar}, \mathrm{He})$ collisions can be well explained.
\end{abstract}

\section{Introduction}

With the advent of powerful heavy-ion accelerators the study of electronic processes initiated by fast highly-ionised heavy projectiles has become experimentally accessible. Until now, electron emission in fast collisions was mainly examined in light systems (Rudd et al 1966, Dettmann et al 1974, Folkmann et al 1975), but recently heavy projectiles have also been used (Breinig et al 1982) in order to investigate the influence of strong perturbing fields. The ionisation of target electrons caused by strong external fields can no longer be described by the first-order Born approximation. This was recognised long ago by Oldham (1967) in connection with the forward peak which is due to electrons that are ejected with a low velocity relative to the projectile. This peak can only be explained if the interaction with the projectile is included to all orders. Macek (1970) performed calculations retaining the first-order terms of the three-body Faddeev expansion (Faddeev 1961), and Band (1974) used a linear combination of atomic orbitals, including a projectile and target continuum state, which reduces to the first order Born theory at high collision velocities. Dettmann et al (1974) pointed out that the forward peak can be described by means of charge transfer to the continuum (CTC) indicating that a higher-order theory is necessary for a quantitative understanding. There have been several higher-order approaches, among them the second-order Born approximation (Dettmann et al 1974, Shakeshaft and Spruch 1978, Miraglia and Ponce 1980), as well as a distorted-wave Born approximation (Salin 1969, Garibotti and Miraglia 1980) where the final electronic state is described by a product function of a projectile and a target eigenstate. In all these theories a high-velocity approximation is introduced to make their application feasible, at the expense of a restricted validity requiring asymptotically large collision velocities.

† Supported by the GSI Darmstadt. 
The calculations also included electron energies outside the forward peak region and, for the symmetric systems studied, there were again found deviations from the first-order Born theory (Salin 1969, Miraglia and Ponce 1980). However, the choice of a projectile eigenstate for the emitted electron, as done in the CTC theories, is only valid as long as the interaction with the projectile dominates. Recently, an approximation based on the Faddeev theory has been suggested which includes a higher-order interaction of the electron with both the projectile and the target, thereby allowing for a projectile as well as a target final electronic state (Jakubassa-Amundsen 1981).

For very asymmetric systems where a large region of the spectrum originates from electrons which move predominantly in the projectile field, the impulse approximation may be applied (McDowell 1961, Briggs 1977), which includes the strong field to all orders and the weak field to first order and has been successfully used for charge transfer into bound states (Jakubassa-Amundsen and Amundsen 1980). This theory includes the second Born approximation but goes beyond it, which will be important for large perturbing fields. For very high collision velocities, the impulse approximation coincides with the second Born theory and thus has the right asymptotic behaviour.

In this paper we apply the semiclassical impulse approximation (SCIA) to continuum charge transfer without introducing a high-velocity approximation. Section 2 contains the theoretical formulation valid for bare projectiles and one-electron target atoms, and in $\S 3$ we extend the theory to arbitrary target atoms. The validity of the SCIA for target ionisation is discussed in $\S 4$, and numerical results are presented in $\S 5$. A short conclusion follows $(\S 6)$. Atomic units are used $(\hbar=m=e=1)$ unless otherwise indicated.

\section{Continuum charge transfer in the impulse approximation}

\subsection{General theory}

Let us consider the ionisation of a one-electron target (with charge $Z_{\mathrm{T}}$ ) by a bare projectile (charge $Z_{\mathrm{P}}$ ). We use the semiclassical theory where the internuclear motion is described by a classical trajectory $\boldsymbol{R}(t)$, as it is somewhat easier to handle than the equivalent wave treatment. Generalising the theory for capture of a target electron by a heavy projectile (Briggs 1977 ) to the capture into the continuum of the projectile, the transition amplitude is in the semiclassical impulse approximation for $Z_{\mathrm{P}} \gg Z_{\mathrm{T}}$ given by

$$
\begin{aligned}
& a_{f i}=-\mathrm{i} \int_{-\infty}^{\infty} \mathrm{d} t \int \mathrm{d} \boldsymbol{q}\left\langle\psi_{f}^{\mathrm{P}}\left|V_{\mathrm{T}}\right| \psi_{q}^{\mathrm{P}}\right\rangle\left\langle\boldsymbol{q} \mid \psi_{i}^{\mathrm{T}}\right\rangle \\
& V_{\mathrm{T}}=-\boldsymbol{Z}_{\mathrm{T}} /|\boldsymbol{r}+\boldsymbol{R}|
\end{aligned}
$$

where $\psi_{f, \boldsymbol{q}}^{\mathrm{P}}$ are projectile continuum states with momentum $\boldsymbol{\kappa}_{f}$ and $\boldsymbol{q}$, respectively, $\psi_{i}^{\mathrm{T}}$ is the initial bound target state, and $V_{\mathrm{T}}$ the target field. The projectile system has been chosen as frame of reference. As we consider fast collisions, the internuclear motion can be described by a straight line trajectory $\boldsymbol{R}=\boldsymbol{b}+\boldsymbol{v} t$ with impact parameter $\boldsymbol{b}$ and collision velocity $\boldsymbol{v}$. Introducing the Fourier transform of $V_{\mathrm{T}}$ and the translational factor of $\psi_{i}^{\mathrm{T}},(2.1)$ can be written as

$$
\begin{aligned}
a_{f i}=\frac{\mathrm{i} \boldsymbol{Z}_{\mathrm{T}}}{2 \pi^{2}} \int_{-\infty}^{\infty} & \mathrm{d} t \int \mathrm{d} \boldsymbol{q} \varphi_{i}^{\mathrm{T}}(\boldsymbol{q}+\boldsymbol{v}) \exp (\mathrm{i} \boldsymbol{q} \cdot \boldsymbol{b}) \int \frac{\mathrm{d} \boldsymbol{s}}{s^{2}} \exp [\mathrm{i} \boldsymbol{s} \cdot(\boldsymbol{b}+\boldsymbol{v} t)] \\
& \times \exp \left[\mathrm{i}\left(\varepsilon_{f}-E_{i}+v^{2} / 2+\boldsymbol{q} \cdot \boldsymbol{v}\right) t\right]\left\langle\psi_{\kappa_{f}}^{\mathrm{P}}(\boldsymbol{r})|\exp (\mathrm{i} \boldsymbol{s} \cdot \boldsymbol{r})| \psi_{\boldsymbol{q}}^{\mathrm{P}}(\boldsymbol{r})\right\rangle
\end{aligned}
$$


where $\varphi_{i}^{\mathrm{T}}(\boldsymbol{q}+\boldsymbol{v})$ is the momentum representation of the target wavefunction, $E_{i}$ is the initial and $\varepsilon_{f}=\kappa_{f}^{2} / 2$ the final electron energy as viewed from the projectile frame. This formulation contains the inelastic scattering matrix element for a projectile continuum state which implies that after ejection into the state $\psi_{q}^{\mathrm{P}}$ by means of the strong projectile field, the electron is scattered by the target field into its final state. The formula is complicated by the fact that three different momenta occur, the momentum of the ejected electron, $\boldsymbol{\kappa}_{f}$, the momentum brought into the collision by means of the target field, $s$, and the momentum of the intermediate scattering state of the electron, $\boldsymbol{q}$.

The time integral in (2.2) can be immediately done, yielding $2 \pi \delta\left(\varepsilon_{f}-E_{i}+v^{2} / 2+\right.$ $(q+s) \cdot v)$. As we are interested in the differential cross section with respect to the electron degrees of freedom, we have to integrate over impact parameter according to

$$
\frac{\mathrm{d}^{2} \sigma}{\mathrm{d} \varepsilon_{f} \mathrm{~d} \Omega_{f}}=\kappa_{f} \int \mathrm{d}^{2} b\left|a_{f i}\right|^{2} .
$$

We now introduce the electron momentum after the action of the target field

$$
\boldsymbol{q}_{1}=\boldsymbol{q}+\boldsymbol{s}
$$

and by means of a variable shift from $\boldsymbol{q}$ to $\boldsymbol{q}_{1}$ the integral over $\boldsymbol{b}$ is easily performed.

We are, however, interested in the double differential cross section in the laboratory frame. Thus we introduce the total momentum transferred to the electron, $\boldsymbol{q}_{2}$, and the final momentum of the electron, $\boldsymbol{k}_{f}$, in the target frame of reference via the relations (as illustrated in figure 1)

$$
\begin{aligned}
& \boldsymbol{k}_{f}=\boldsymbol{\kappa}_{f}+\boldsymbol{v} \\
& \boldsymbol{q}_{2}=\boldsymbol{q}_{1}+\boldsymbol{v}-\boldsymbol{k}_{f}
\end{aligned}
$$

and define the final electron energy $E_{f}=k_{f}^{2} / 2$. For computational convenience we also transform the coordinate $s$ to the variable $s_{0}$ (which is identical to the old variable $q$ ) by means of $s_{0}=q_{1}-s$. Then we obtain together with $(2.4 b)$ :

$$
\begin{aligned}
\frac{\mathrm{d}^{2} \sigma}{\mathrm{d} E_{f} \mathrm{~d} \Omega_{f}}=\frac{4 k_{f} \boldsymbol{Z}_{\mathrm{T}}^{2}}{v} \int \mathrm{d} \boldsymbol{q}_{2} \delta\left(\boldsymbol{q}_{2} \cdot \boldsymbol{v}+k_{f}^{2} / 2-E_{i}\right) \\
\quad \times\left|\int \frac{\mathrm{d} s_{0}}{\left(\boldsymbol{q}_{2}+\boldsymbol{k}_{f}-\boldsymbol{v}-\boldsymbol{s}_{0}\right)^{2}} \boldsymbol{\varphi}_{i}^{\mathrm{T}}\left(\boldsymbol{s}_{0}+\boldsymbol{v}\right)\left\langle\psi_{\boldsymbol{k}_{f}-v}^{\mathrm{P}}\left|\exp \left[\mathrm{i}\left(\boldsymbol{q}_{2}+\boldsymbol{k}_{f}-\boldsymbol{v}-\boldsymbol{s}_{0}\right) \cdot \boldsymbol{r}\right]\right| \psi_{\mathbf{s}_{0}}^{\mathrm{P}}\right\rangle\right|^{2} .
\end{aligned}
$$

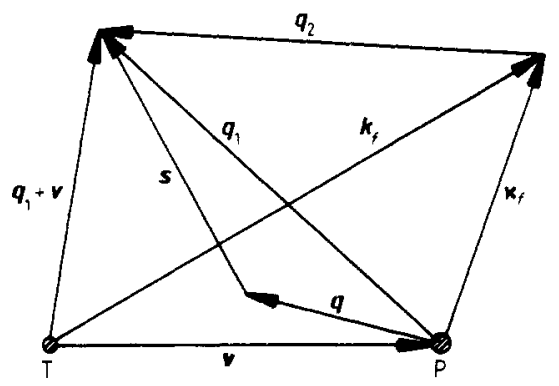

Figure 1. Relations between the electron momenta as viewed from the projectile and the target frame, respectively. 
When Coulomb waves are inserted for $\psi_{\boldsymbol{k}_{f} \rightarrow v}^{\mathrm{P}}$ and $\psi_{s_{0}}^{\mathrm{P}}$, the scattering matrix element can be calculated analytically, as it can be expressed as a derivative of a matrix element evaluated by Nordsieck (1954)

$$
\begin{aligned}
\left\langle\psi_{\boldsymbol{\kappa}_{f}}^{\mathrm{P}}|\exp (\mathrm{is} \cdot \boldsymbol{r})| \psi_{s_{0}}^{\mathrm{P}}\right\rangle & =\lim _{\varepsilon \rightarrow 0}\left(-\frac{\mathrm{d}}{\mathrm{d} \varepsilon}\right) \int \mathrm{d} \boldsymbol{r} \psi_{\boldsymbol{\kappa}_{f}}^{* \mathrm{P}}(\boldsymbol{r}) r^{-1} \exp (\mathrm{i} \boldsymbol{s} \cdot \boldsymbol{r}-\varepsilon r) \psi_{s_{0}}^{\mathrm{P}}(\boldsymbol{r}) \\
= & -(2 \pi)^{-2} \exp \left(\pi \eta_{f} / 2\right) \Gamma\left(1-\mathrm{i} \eta_{f}\right) \exp \left(-\pi \eta_{0} / 2\right) \Gamma\left(1-\mathrm{i} \eta_{0}\right) \alpha^{-1} M\left(\boldsymbol{q}_{2}, \boldsymbol{s}_{0}\right) \\
M\left(\boldsymbol{q}_{2}, s_{0}\right)= & \alpha^{\mathrm{i} \eta_{0}} \gamma^{\mathrm{i} \eta_{f}-\mathrm{i} \eta_{0}-1}(\gamma+\delta)^{-\mathrm{i} \eta_{f}-1}\left[{ }_{2} F_{1}\left(1-\mathrm{i} \eta_{0}, \mathrm{i} \eta_{f}, 1 ; \frac{\alpha \delta-\beta \gamma}{\alpha(\gamma+\delta)}\right)\right. \\
& \times\left[\eta_{f}\left(\kappa_{f} \gamma-s_{0} \delta\right)+\eta_{0} s_{0}(\gamma+\delta)\right]+{ }_{2} F_{1}\left(2-\mathrm{i} \eta_{0}, 1+\mathrm{i} \eta_{f}, 2 ; \frac{\alpha \delta-\beta \gamma}{\alpha(\gamma+\delta)}\right) \\
& \left.\times \frac{\left(1-\mathrm{i} \eta_{0}\right) \gamma \eta_{f}}{\alpha(\gamma+\delta)}\left[s_{0} \delta(\alpha+\beta)-\kappa_{f} \gamma(\alpha+\beta+\gamma+\delta)\right]\right]
\end{aligned}
$$

where ${ }_{2} F_{1}$ is a hypergeometric function, $\Gamma$ the gamma function, $\eta_{f}=Z_{\mathrm{P}} / \kappa_{f}, \eta_{0}=Z_{\mathrm{P}} / s_{0}$ and we have introduced the abbreviations

$$
\begin{aligned}
& \alpha=q_{2}^{2} / 2 \quad \beta=\boldsymbol{\kappa}_{f} \cdot \boldsymbol{q}_{2}-\mathrm{i} \boldsymbol{\varepsilon} \\
& \gamma=\boldsymbol{s}_{0} \cdot \boldsymbol{q}_{2}-q_{2}^{2} / 2+\mathrm{i} \varepsilon \quad \delta=\boldsymbol{\kappa}_{f} s_{0}+\boldsymbol{\kappa}_{f} \cdot\left(\boldsymbol{s}_{0}-\boldsymbol{q}_{2}\right)+\mathrm{i} \varepsilon \quad \varepsilon \rightarrow 0
\end{aligned}
$$

\subsection{Peaking approximation}

Unless $\boldsymbol{\kappa}_{f}=\boldsymbol{k}_{f}-\boldsymbol{v}$ is parallel to $\boldsymbol{v}$ none of the five nontrivial integrals of (2.5) can be performed analytically, due to the complicated structure of $M$. In order to do any numerical evaluation of (2.5) we have to introduce a peaking approximation, in a similar way as we have done for bound-state charge transfer (Jakubassa-Amundsen and Amundsen 1980). As the Fourier transform $\varphi_{i}^{\mathrm{T}}\left(s_{0}+v\right)$ is strongly peaked at $s_{0}=-v$ the main contribution to the integral over $s_{0}$ comes from the region around $-v$. Keeping in mind that the momentum transfer is mainly along the direction of $v$ (which we take as $z$ axis), we replace $s_{0}$ by $s_{0 z} e_{z}$ in the matrix element, thus putting the momentum components $s_{0 \perp}$ perpendicular to $v$ equal to zero while allowing for a variation of the $z$ component around $-v$. This approximation is better the smaller $v_{i} / v$ is, where $v_{i}$ is the electron orbiting velocity in the initial state. Note that, in contrast to the bound-state charge transfer, a peaking approximation where $s_{0}$ is set equal to $-\boldsymbol{v}$ is not possible, as this would introduce a non-integrable singularity at $\gamma=0$.

With the above approximation, the integration over the momentum components $\boldsymbol{s}_{0 \perp}$ can be performed. For a target $1 \mathrm{~s}$ state, it yields

$$
\begin{array}{r}
\int_{0}^{\infty} s_{0 \perp} \mathrm{d} s_{0 \perp} \int_{0}^{2 \pi} \mathrm{d} \varphi_{s_{0}} \varphi_{i}^{\mathrm{T}}\left(\boldsymbol{s}_{0}+\boldsymbol{v}\right) \frac{1}{\left(\boldsymbol{q}_{2}+\boldsymbol{k}_{f}-\boldsymbol{v}-\boldsymbol{s}_{0}\right)^{2}}=2^{3 / 2} Z_{\mathrm{T}}^{5 / 2} f_{0}\left(\boldsymbol{q}_{2}, s_{0 z}\right) \\
f_{0}\left(\boldsymbol{q}_{2}, s_{0 z}\right)=\frac{1}{\beta_{0}}\left(\frac{q_{0}^{2}+p_{\perp}^{2}}{p_{0}^{2}}-1\right)-\frac{\alpha_{0}}{2 \beta_{0}^{3 / 2}} \ln \frac{2 \sqrt{\beta_{0}}\left(q_{0}^{2}+p_{\perp}^{2}\right)+2 \beta_{0}+\alpha_{0} p_{0}^{2}}{p_{0}^{2}\left(\alpha_{0}+2 \sqrt{\beta_{0}}\right)}
\end{array}
$$

with

$$
p_{0}^{2}=Z_{\mathrm{T}}^{2}+\left(s_{0 z}+v\right)^{2} \quad q_{0}^{2}=\left(q_{2 z}+k_{f z}-v-s_{0 z}\right)^{2}
$$




$$
\begin{aligned}
& p_{\perp}^{2}=\left(\boldsymbol{q}_{2}+\boldsymbol{k}_{f}\right)^{2}-\left(q_{2 z}+k_{f z}\right)^{2} \\
& \alpha_{0}=2\left(q_{0}^{2}-p_{\perp}^{2}-p_{0}^{2}\right) \quad \beta_{0}=\left(q_{0}^{2}+p_{\perp}^{2}-p_{0}^{2}\right)^{2}+4 p_{\perp}^{2} p_{0}^{2} .
\end{aligned}
$$

Then (2.5) can be written in the form

$$
\begin{aligned}
\frac{\mathrm{d}^{2} \sigma}{\mathrm{d} E_{f} \mathrm{~d} \Omega_{f}}=\frac{32}{\pi^{3}} & \frac{k_{f} Z_{\mathrm{T}}^{7}}{v^{2}} \frac{\eta_{f}}{1-\exp \left(-2 \pi \eta_{f}\right)} \int_{q_{\min }}^{\infty} \frac{\mathrm{d} q_{2}}{q_{2}^{3}} \int_{0}^{\pi} \mathrm{d} \varphi_{q} \\
& \times\left|\int_{-\infty}^{\infty} \mathrm{d} s_{0 z} f_{0}\left(\boldsymbol{q}_{2}, s_{0 z}\right) \exp \left(-\pi \eta_{0} / 2\right) \Gamma\left(1-\mathrm{i} \eta_{0}\right) M\left(\boldsymbol{q}_{2}, s_{0 z} \boldsymbol{e}_{z}\right)\right|^{2}
\end{aligned}
$$

where $q_{\min }=-q_{2 z}=\left(k_{f}^{2} / 2-E_{i}\right) / v$. In order to get convergence, it is sufficient to integrate over a small region of $s_{0 z}$ around $-v$. The angle $\varphi_{q}$ appears in the product $\boldsymbol{q}_{2} \cdot \boldsymbol{k}_{f}=k_{f}\left(q_{2 z} \cos \vartheta_{f}+\left(q_{2}^{2}-q_{2 z}^{2}\right)^{1 / 2} \sin \vartheta_{f} \cos \varphi_{q}\right)$ which is contained in both $f_{0}$ and $M$. If the electron is ejected parallel to the beam direction $\left(\vartheta_{f}=0^{\circ}, 180^{\circ}\right)$, the dependence on $\varphi_{q}$ disappears and the $\varphi_{q}$ integral is replaced by $\pi$.

\subsection{Properties of the scattering matrix element and the cross section}

The structure of the energy distribution of the emitted electrons can be extracted from (2.9) without further evaluation. For this aim we look for conditions for the momenta $s_{0}$ and $q_{2}$ where the integrand becomes very large. The matrix element $M\left(q_{2}, s_{0}\right)$ is singular for $\gamma=0$. Together with the choice $s_{0}=-v$ where $\varphi_{i}^{\mathrm{T}}$ is peaked, it follows from (2.7)

$$
\gamma=-v \cdot q_{2}-q_{2}^{2} / 2=0
$$

The $z$. component of $q_{2}$ is determined by the energy conservation such that we have

$$
-v q_{2 z}=k_{f}^{2} / 2-E_{i}=q_{2}^{2} / 2=\frac{q_{2 z}^{2}}{2 \cos ^{2} \vartheta_{q}}
$$

where we have introduced the polar angle $\vartheta_{q}$ of $\boldsymbol{q}_{2}$. In order to obtain an equation determining $k_{f}$, i.e. to find $\vartheta_{q}$, we use the fact that the Fourier transform of $V_{\mathrm{T}}$ enhances small momentum components $s$. From (2.5) it follows (with $s_{0}=-v$ ) that for fixed $q_{2}, s$ has its smallest value when $q_{2}$ has the opposite direction of $\boldsymbol{k}_{f}$ :

$$
\boldsymbol{q}_{2}=-c_{0} \boldsymbol{k}_{f} \quad c_{0}>0
$$

which means that $\cos \vartheta_{q}=-\cos \vartheta_{f}$. Inserting this into $(2.11)$ we find the desired equation

$$
2 v^{2} \cos ^{2} \vartheta_{f}=k_{f}^{2} / 2-E_{i}
$$

For $E_{i} \ll v^{2} / 2$ we can neglect $E_{i}$ in the above equation if $k_{f}$ is of the order of $v$, and obtain $k_{f}=2 v \cos \vartheta_{f}$. Thus we find again the binary encounter peak from the first-order theory.

In addition to this binary encounter peak the differential cross section shows the forward peak at $\eta_{f}=\infty$, i.e. $k_{f}=v$ which arises from the fact that the final state has been chosen as eigenstate to the projectile. There is an interesting phenomenon connected with the forward peak. Even if one removes the divergent normalisation factor of $\psi_{\kappa f}^{\mathrm{P}}$, there remains a discontinuity at $k_{f}=v$ for $\vartheta_{f}=0$ in contrast to the first Born approximation. This has been predicted by Lucas et al (1980) from the assumption of continuity of the capture amplitudes across the ionisation threshold of the 
projectile. However, from this argument it is not clear why the first-order BrinkmanKramers theory for capture (where $\psi_{s_{0}}^{\mathrm{P}}$ in (2.5) is replaced by a plane wave), although dependent on $\boldsymbol{\kappa}_{f}$ in an asymmetric way, does not show this phenomenon.

The discontinuity originates from the matrix element $M\left(q_{2}, s_{0}\right)$. In.order to demonstrate this, we put $s_{0} \simeq-v$ and $q_{2} \simeq q_{2 z} e_{z}$ where the main contribution to the integrals comes from. Then it follows from (2.7) that $\gamma \simeq 3 v^{2} / 8>0$, while $\beta \simeq-v \kappa_{f} / 2, \delta \simeq v \kappa_{f} / 2$ if $k_{f}>v$ and $\beta \simeq v \kappa_{f} / 2, \delta \approx 3 v \kappa_{f} / 2$ if $k_{f}<v$ where we retained only the leading terms in $\kappa_{f}$. This means that $\beta$ and $\delta$ go simultaneously to zero for $k_{f}=v$. When $\delta \rightarrow 0$ and $\beta \rightarrow 0$ for $\eta_{f} \rightarrow \infty$, the hypergeometric functions in (2.6) may be reduced according to

$$
\begin{aligned}
& { }_{2} F_{1}\left(1-\mathrm{i} \eta_{0}, \mathrm{i} \eta_{f}, 1 ; \frac{\alpha \delta-\beta \gamma}{\alpha(\gamma+\delta)}\right) \rightarrow{ }_{1} F_{1}\left(1-\mathrm{i} \eta_{0}, 1 ; x\right) \\
& x=\mathrm{i} \eta_{f} \frac{\alpha \delta-\beta \gamma}{\alpha \gamma} \simeq \begin{cases}\mathrm{i} Z_{\mathrm{P}} \frac{v(\alpha+\gamma)}{2 \alpha \gamma} \simeq \frac{\mathrm{i} 16 Z_{\mathrm{P}}}{3 v} & k_{f}>v \\
\mathrm{i} Z_{\mathrm{P}} \frac{v(3 \alpha-\gamma)}{2 \alpha \gamma} \simeq 0 & k_{f}<v .\end{cases}
\end{aligned}
$$

Thus, the hypergeometric function is approximately 1 for $k_{f} \rightarrow v-0$, while it has a different value when $k_{f}$ approaches $v$ from above. In addition, there is a discontinuity in the phase because

$$
(\gamma+\delta)^{-\mathrm{i} \eta_{f}} \simeq \exp \left[-\mathrm{i} \eta_{f}(\ln \gamma+\delta / \gamma)\right]
$$

is different for $k_{f}>v$ and $k_{f}<v$. For $\vartheta_{f} \neq 0, \eta_{f}$ is finite and the zeros of $\beta$ and $\delta$ do not coincide independently of the integration variables, such that the matrix element is continuous in that case.

As has been pointed out by Lucas et al (1980) the discontinuity may be understood by means of a transformation to the projectile frame. In this frame, electrons with $k_{f}<v$ are ejected into the backward direction (antiparallel to $v$, i.e. in the same direction as the target motion). Electrons with $k_{f}>v$ are ejected with a lower intensity, as they move in the opposite direction from the target (i.e. parallel to $v$ ).

\section{CTC from a screened target}

If the target has more than one electron, both the initial electronic wavefunction and the target potential have to be modified. These modifications can easily be incorporated into our theory if the single particle approximation is used, implying that the motion of the active electron is described by an effective potential. The potential of an electron in the field of the target ion of (ionic) charge $Z_{i}$ may be approximated by (Nielsen and Dahler 1977)

$$
V_{\mathrm{T}}(r)=-\frac{Z_{\mathrm{T}}-Z_{\mathrm{i}}}{r} \exp (-r / d)\left(1+\frac{r}{2 d}\right)-\frac{Z_{\mathrm{i}}}{r}
$$

where the parameter $d$ is determined such that the expectation value of the Hamiltonian $H_{\mathrm{T}}=-\Delta / 2+V_{\mathrm{T}}(r)$ equals the experimental binding energy of the electron under consideration. 
The electronic wavefunction may be expressed as a Slater determinant of oneelectron orbitals, the space parts of which have the form (Clementi and Roetti 1974)

$$
\psi_{i}(\boldsymbol{r})=\sum_{i=1}^{n} c_{i} \exp \left(-\xi_{i} r\right) Y_{l m}(\hat{\boldsymbol{r}})
$$

where $l$ and $m$ denote the angular momentum quantum numbers of the initial state, and the parameters $c_{i}, \xi_{i}$ and $n(i=1, \ldots, n)$ are determined from an analytical fit to a Hartree-Fock calculation. In the case of a helium target they are given in the appendix.

The Fourier transforms of the wavefunction and the potential enter into the evaluation of the transition amplitude (2.3)

$$
\begin{aligned}
\varphi_{i}^{\mathrm{He}}(\boldsymbol{q}) & =\frac{\sqrt{2}}{\pi} \sum_{i=1}^{5} c_{i} \xi_{i} \frac{1}{\left(\xi_{i}^{2}+q^{2}\right)^{2}} \\
V^{\mathrm{He}}(s) & =-\frac{\sqrt{2}}{\sqrt{\pi}}\left(\frac{1}{s^{2}}+\frac{1}{s^{2}+d^{-2}}+\frac{1}{d^{2}\left(s^{2}+d^{-2}\right)^{2}}\right) .
\end{aligned}
$$

The doubly differential cross section for electron emission can still be obtained from (2.9.) with $Z_{\mathrm{T}}$ set equal to 1 and $f_{0}\left(q_{2}, s_{0 z}\right)$, i.e. the integral in (2.8), replaced by $f_{0}^{\mathrm{He}}\left(\boldsymbol{q}_{2}, s_{0 z}\right)$ which is derived in the appendix.

The effect of using a screened, i.e. weaker, target field is generally a lowering of the cross section compared with the unscreened case, while the application of a modified wavefunction may have effects in both directions, depending on the magnitude of the transferred momentum. Near the forward peak, i.e. for the production of electrons with energy of about $v^{2} / 2$, a large momentum has to be transferred, which implies that the small-r behaviour of $\psi_{i}^{\mathrm{T}}$ enters the cross section. In the region of the binary encounter peak, however, the transferred momentum may be very small such that $\psi_{i}^{\mathrm{T}}$ at large $r$ gives the dominant contribution. In the case of a He target, for example, a hydrogenic wavefunction with an effective charge of 1.75 can reproduce the forward peak intensity quite well. Note that for fast collisions the influence of the screening of the target field amounts to only a few per cent for $E_{f} \gg\left|E_{i}\right|$, as only the high Fourier components of $V_{\text {T }}$ play a role.

\section{Validity of the impulse approximation}

The impulse approximation (2.2) can be derived from the Faddeev theory (Faddeev 1961) which provides an expansion of the exact two-potential scattering state $\psi_{f}$

$\left\langle\psi_{f}^{-}\right|=\left\langle\psi_{0}\right|\left(1+V_{\mathrm{T}} G_{\mathrm{T}}+V_{\mathrm{P}} G_{\mathrm{P}}+V_{\mathrm{P}} G_{\mathrm{P}} V_{\mathrm{T}} G_{\mathrm{T}}+V_{\mathrm{T}} G_{\mathrm{T}} V_{\mathrm{P}} G_{\mathrm{P}}+V_{\mathrm{P}} G_{\mathrm{P}} V_{\mathrm{T}} G_{\mathrm{T}} V_{\mathrm{P}} G_{\mathrm{P}}+\ldots\right)$

in terms of the electron Green's functions $G_{\mathrm{T}, \mathrm{P}}$ of the target and projectile acting on a plane wave $\psi_{0}$. If all but the first-order terms in the weak target field $V_{\mathrm{T}}$ are neglected in (4.1), and this approximation for $\psi_{f}^{-}$inserted into the transition amplitude

$$
a_{f i}=-\mathrm{i} \int_{-\infty}^{\infty} \mathrm{d} t\left\langle\psi_{f}^{-}\left|V_{\mathbf{P}}\right| \psi_{i}^{\mathrm{T}}\right\rangle
$$

one obtains an equation of type (2.2) with an off-shell continuum wavefunction in place of $\psi_{q}^{\mathrm{P}}$ (Jakubassa-Amundsen and Amundsen 1980). Thus the SCIA theory (2.2) involves two approximations, retaining only first-order terms in $V_{\mathrm{T}}$ and going on-shell. 
As shown in Jakubassa-Amundsen (1981) the omission of higher-order terms is only possible as long as the motion of the electron is governed by the strong potential $V_{\mathrm{P}}$. If the electron moves slowly with respect to the target $\left(k_{f} \rightarrow 0\right)$, it will be in a target eigenstate which is included in (4.1) but not in (2.1). A target eigenstate at low energy is characterised by the divergence of its normalisation constant as $k_{f}^{-1 / 2}$ which mirrors the strong attraction and thus multiple interaction between electron and target nucleus. A theory like the impulse approximation which does not include the target field to all orders can not reproduce the $k_{f}^{-1}$ behaviour of the differential cross section $\mathrm{d} \sigma / \mathrm{d} k_{f}$, and therefore does not lead to a non-zero value for $\mathrm{d} \sigma / \mathrm{d} E_{f}$ at $k_{f}=0$, as it should.

However, for very asymmetric collision systems the range of validity of (2.1) extends to rather small values of $k_{f}$. For an estimate, we apply a classical picture and assume that the impulse approximation ceases to be valid when the potential energy of the electron in the projectile and target field becomes equal. As shown in figure 2, the electron which is ejected with momentum $k_{f}$ has moved a distance $k_{f} \tau$ after time $\tau$, while the projectile has moved a distance $v \tau$ away from the target (for $b=0$ ).

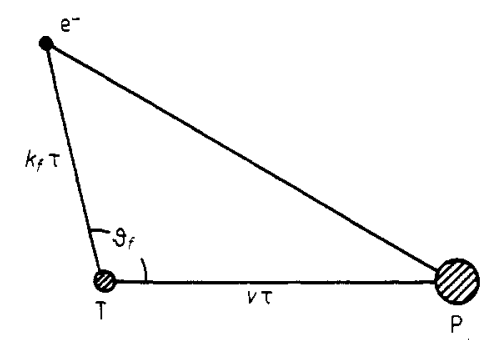

Figure 2. Coordinate system showing the distances of the electron and the projectile from the target at time $\tau$ after the collision.

Equating the electron's energy in the projectile and target field, we find

$$
\frac{Z_{\mathbf{T}}}{k_{f} \tau}=\frac{Z_{\mathrm{P}}}{\tau\left[\left(v-k_{f} \cos \vartheta_{f}\right)^{2}+k_{f}^{2} \sin ^{2} \vartheta_{f}\right]^{1 / 2}}
$$

For asymmetric systems $\left(Z_{\mathrm{T}} / Z_{\mathrm{P}} \ll 1\right)$ this leads, independently of the angle $\vartheta_{f}$, to the condition

$$
k_{f} \geqslant \frac{Z_{\mathrm{T}}}{Z_{\mathrm{P}}} v
$$

which excludes only the low-velocity part of the electron spectrum. Note, however, that (2.1) fails to describe the total cross sections as they are dominated by this region.

The effect of off-shell wavefunctions has been investigated for charge transfer to bound states, where the exact off-shell function is approximated by the Coulomb wave $\psi_{q}^{\mathrm{P}}$ times a constant normalisation factor (Okubo and Feldman 1960, JakubassaAmundsen and Amundsen 1981, Macek and Alston 1982). The corrections thereby introduced are at most of the order of $v_{\mathrm{e}} / v$ for $v \geqslant v_{\mathrm{e}}$, where $v_{\mathrm{e}}$ is the electron orbiting velocity of the heavy particle (projectile) bound state, and they are no larger for $\mathrm{L}$-shell than for $\mathrm{K}$-shell capture. As $v_{\mathrm{e}} / v$ decreases when the continuum threshold is approached, one expects that the off-shell effects may be neglected for continuum charge transfer. This is supported by the fact that the off-shell function is, like the Coulomb wave, asymptotically normalised to a plane wave. 
When the off-shell function is approximated in the same way as for bound-state charge transfer, the integral over the momentum $s_{0 \perp}$ ( $\operatorname{cf}(2.8)$ ) has to be done numerically. We have perforined test calculations for the case of $\vartheta_{f}=0$ and found that the on-shell results were overestimated in the region of the forward peak by $20-60 \%$; however, much more around the binary encounter peak. The failure of the simple renormalisation to describe the off-shell correction for CTC may be explained by recalling that considerable contributions to the scattering matrix element $\left\langle\psi_{f}^{\mathrm{P}}\left|V_{\mathrm{T}}\right| \psi_{q}^{\mathrm{P}}\right\rangle$ come from large $r$, where the off-shell function is not correctly described by a renormalised Coulomb wave.

\section{Numerical results and comparison with other theories and experiment}

We have evaluated the doubly differential cross section for electron emission in $(\mathrm{Kr}, \mathrm{H})$ and $(\mathrm{Ar}, \mathrm{He}$ ) collisions at $20 \mathrm{MeV} / N$ and $8.18 \mathrm{MeV} / N$, respectively. The numerical evaluation of the integrals in (2.9) is rather tricky and we point out the main difficulties. As mentioned above, one can safely avoid dealing with the singular and rapidly oscillating integrand at $s_{0 z}=0$ (where $\eta_{0} \rightarrow \infty$ ) by restricting the integration limits to values close to $-v$; the choice of $s_{\min } \simeq-2 v$ and $s_{\max } \simeq-v / 4$ is sufficient for a good convergence. Further, the matrix element diverges for $\gamma=0$, i.e. $s_{0 z} \equiv \bar{s}_{0}=$ $-q_{2}^{2} /\left(2 q_{\text {min }}\right)$ like $\gamma^{\mathrm{i} \eta_{f}-\mathrm{i} n_{0}-1}$; this may be circumvented by separating the divergent part from the slowly varying rest of the integrand and treating it analytically:

$$
\begin{aligned}
\int_{s_{\min }}^{s_{\max }} \mathrm{d} s_{0 z} & \gamma^{\mathrm{i} \eta_{f}-\mathrm{i} \bar{\eta}_{0}-1} F\left(s_{0 z}\right) \\
= & -\left.\frac{F\left(\bar{s}_{0}\right)}{\mathrm{i}\left(\eta_{f}-\bar{\eta}_{0}\right) q_{\min }}\left(-s_{0 z} q_{\min }-\frac{1}{2} q_{2}^{2}+\mathrm{i} \varepsilon\right)^{\mathrm{i}\left(\eta_{f}-\bar{\eta}_{0}\right)}\right|_{s_{\min }} ^{s_{\max }} \\
& +\int_{s_{\min }}^{s_{\max }} \mathrm{d} s_{0 z} \gamma^{\mathrm{i} \eta_{f}-\mathrm{i} \bar{\eta}_{0}-1}\left(F\left(s_{0 z}\right)-F\left(\bar{s}_{0}\right)\right)
\end{aligned}
$$

where $\bar{\eta}_{0}=\eta_{0}\left(\bar{s}_{0}\right)$. Near the forward peak where $\eta_{f}$ becomes very large, the remaining integral is still badly behaved. We performed a coordinate transformation which can be applied when the integrand has a branch point singularity (Wadehra et al 1981):

$$
\begin{array}{ll}
s_{0 z}<\bar{s}_{0} & s_{0 z}=-x^{2} / q_{\min }+\bar{s}_{0} \\
s_{0 z}>\bar{s}_{0} & s_{0 z}=x^{2} / q_{\min }+\bar{s}_{0}
\end{array}
$$

which makes the integrand zero at $\gamma=0$. Further we used an expansion of the hypergeometric functions in the matrix element for $|\gamma|<0.1$ (and $|\gamma /(\gamma+\delta)|<0.3$ ) up to first order in $\gamma$. Otherwise, these functions are calculated by means of their series representations and analytical continuations, respectively (Abramowitz and Stegun 1965). For the evaluation of the integral over the angle $\varphi_{q}$ (for $\vartheta_{f}>0$ ) it is important that in the region of the binary encounter peak the integrand is sharply peaked at $\varphi_{q}=\pi$ as, according to $(2.12), q_{2}$ has preferably the opposite direction of $\boldsymbol{k}_{f}$ (which lies in the plane $\varphi=0$ ). We estimate the accuracy of our calculations to be about $5 \%$.

Figure 3 shows the electron spectrum at $\vartheta_{f}=0$ for the ionisation of $\mathrm{H}$ by $\mathrm{Kr}$. Due to the high collision velocity, the binary encounter peak extends over many orders of magnitude. At $E_{f}=10.9 \mathrm{keV}$, the forward cusp is seen, and at very low energies, the 


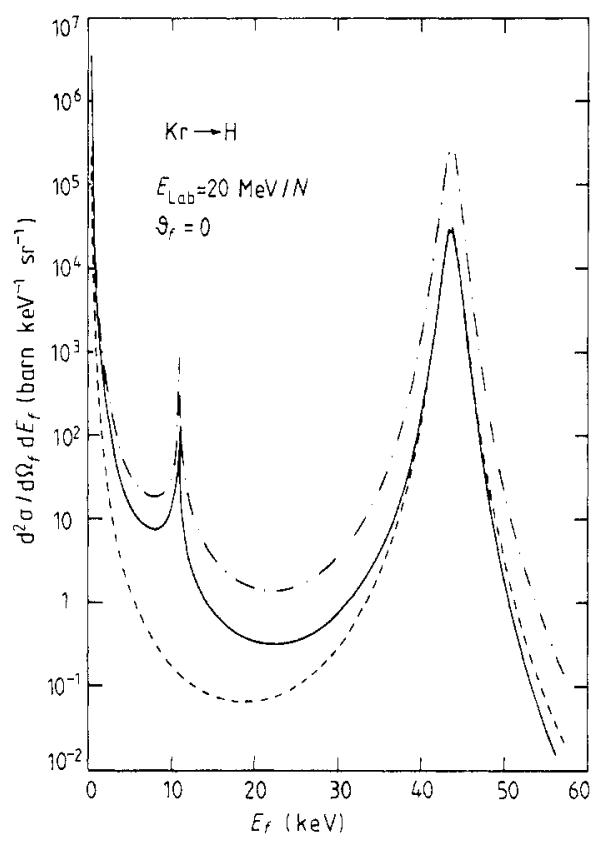

Figure 3. Cross section for electron emission in collisions of $\mathrm{Kr}$ with $\mathrm{H}$ at a collision velocity of $v / \mathrm{c}=$ 0.2065 as a function of electron energy at zero emission angle. The full curve denotes the sciA theory (equation (2.9)), the broken curve is the first Born theory and the chain curve is the MiragliaPonce approximation (equation (5.4)).

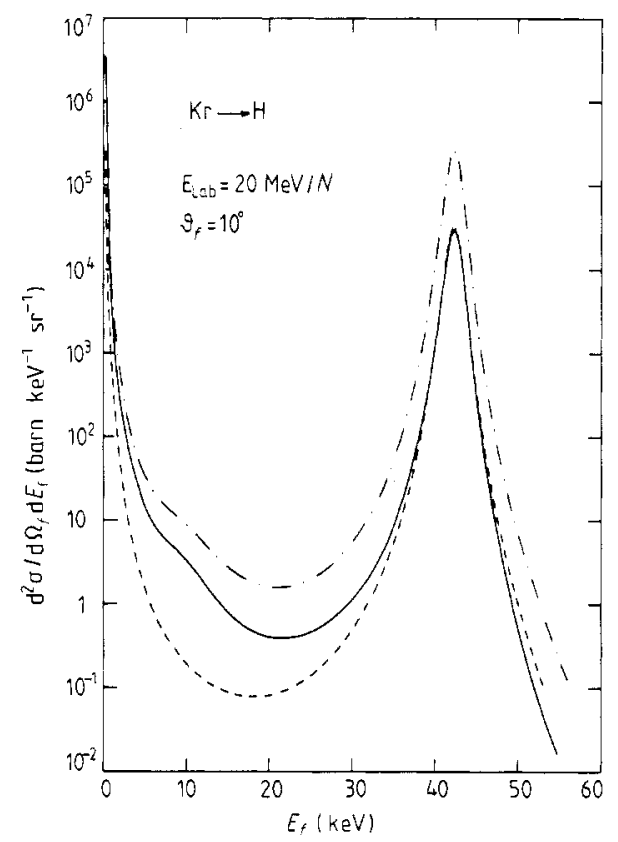

Figure 4. As figure 3 but for emission angle $\vartheta_{f}=10^{\circ}$.

cross section shows a steep rise, indicating that the main contribution to the total cross section comes from this region. We have compared our results with the first-order Born theory

$$
\frac{\mathrm{d}^{2} \sigma^{\mathrm{B}}}{\mathrm{d} E_{f} \mathrm{~d} \Omega_{f}}=\frac{4 Z_{\mathrm{P}}^{2} k_{f}}{v} \int \frac{\mathrm{d} \boldsymbol{q}}{q^{4}} \delta\left(E_{f}-E_{\mathrm{i}}-\boldsymbol{q} \cdot \boldsymbol{v}\right)\left|\left\langle\psi_{f}^{\mathrm{T}}|\exp (\mathrm{i} \boldsymbol{q} \cdot \boldsymbol{r})| \psi_{i}^{\mathrm{T}}\right\rangle\right|^{2}
$$

and the formula of Miraglia and Ponce (1980)

$$
\frac{\mathrm{d}^{2} \sigma^{\mathrm{MP}}}{\mathrm{d} E_{f} \mathrm{~d} \Omega_{f}}=\frac{2 \pi \eta_{f}}{1-\exp \left(-2 \pi \eta_{f}\right)} \frac{\mathrm{d}^{2} \sigma^{\mathrm{B}}}{\mathrm{d} E_{f} \mathrm{~d} \Omega_{f}}
$$

which includes higher-order contributions from the projectile field by means of the normalisation constant of the projectile wavefunction, and compares well with the second Born approximation for symmetric systems. As seen from figure 3, the theory (5.4) explains the forward peak, but otherwise overestimates the cross section considerably. While the first Born approximation fails in the region $k_{f} \approx v$, it describes the binary encounter peak very well. This may be explained by recalling that this peak has a classical origin, as its position corresponds to the maximum energy which can be transferred to an electron at rest in a binary collision with the projectile. Any correct quantum mechanical theory should thus give a result close to the binary encounter approximation, which already holds for the first Born theory. Further, the shape of the peak is given by the momentum distribution of the target electron in its 
initial state, and should therefore be independent of the kind of interaction with the projectile one includes in the theory. The behaviour of the differential cross section is very similar if the emission angle is increased to $10^{\circ}$ (figure 4 ). However, for this angle, the forward peak has nearly disappeared.

In order to make the differences to the other theories more visible, we have plotted in figure 5 the cross section ratios between the impulse approximation and the Miraglia-Ponce formula in the forward peak region. As the Coulomb wave normalisation is divided out, the discontinuity of the SCIA cross section for $\vartheta_{f}=0$ at $k_{f}=v$ is clearly seen. It leads to a large asymmetry of the forward peak, with a much higher intensity on the low-energy side. Note that this is in contrast to the shape of the forward peak produced by projectile electrons, where the high-energy side is enhanced (Jakubassa-Amundsen 1981). The discontinuity is not only visible in the cross section, but also in the structure of the $q_{2}$-dependent integrand (after the $s_{0 z}$ integral is done). The value $q_{\mathrm{m}}$, where the integrand has its largest value, is much greater than $q_{\min }$ if $k_{f}<v$, increasing slightly when $k_{f} \lesseqgtr v$. For $k_{f}>v, q_{\mathrm{m}}$ is always equal to $q_{\min }$ (which increases monotonically with $k_{f}$ ) such that a plot of $q_{m}$ against $E_{f}$ has a similar shape to the $\vartheta_{f}=0$ curve. For $\vartheta_{f}$ slightly larger than 0 , the values drop in a narrow region when $k_{f}$ is increased beyond $v$. For angles $\vartheta_{f} \geqslant 10^{\circ}$, the asymmetry with respect to $k_{f}=v$ becomes very weak.

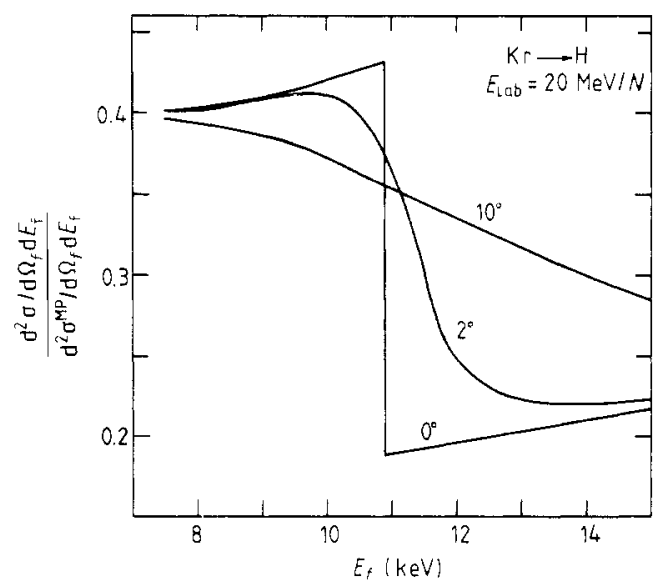

Figure 5. Cross section ratio for electron emission in $(\mathrm{Kr}, \mathrm{H})$ collisions at $20 \mathrm{MeV} / N$ calculated with the impulse approximation and the Miraglia-Ponce approximation (MP). The ratio is shown as a function of electron energy for various emission angles $\vartheta_{f}$.

Figure 6 shows the ratio of the cross sections calculated with the impulse approximation and the Born theory in the region of the binary encounter peak. It is seen that the ratio is approximately one near the peak energy $E_{\mathrm{m}}=2 v^{2} \cos ^{2} \vartheta_{f}$, a little hump marking its position at the different emission angles $\vartheta_{f}$. There is also a slight asymmetry introduced by the higher-order theory, enhancing the binary encounter peak at the low-energy side. At higher energies, the Born theory overestimates the impulse approximation, indicating that even at this collision velocity the projectile influence is non-negligible for $E_{f}>E_{\mathrm{m}}$ (note that $Z_{\mathrm{P}} / 2 v=0.64$ ).

We also performed calculations in the forward peak region for the ( $\mathrm{Ar}, \mathrm{He}$ ) system, where experimental data are available (Breinig et al 1982). In order to compare with 


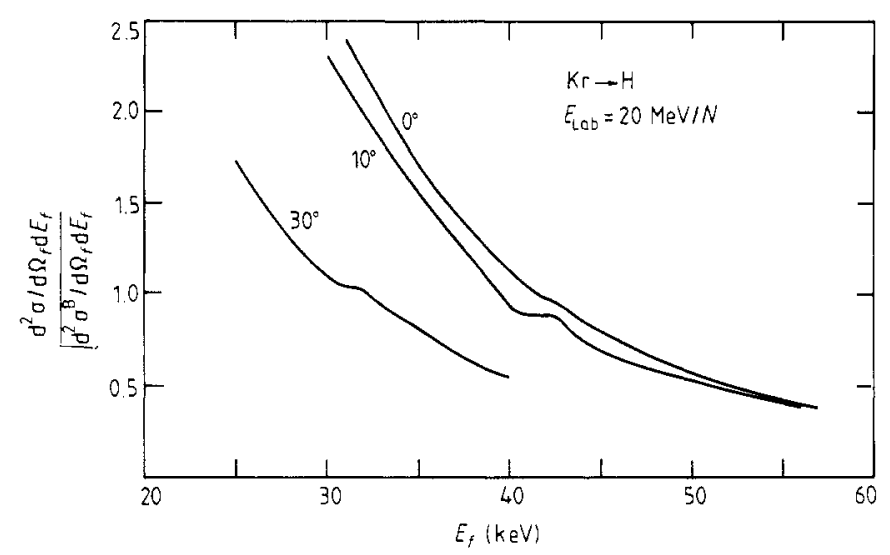

Figure 6. Cross section ratio for electron emission in $(\mathrm{Kr}, \mathrm{H})$ collisions at $20 \mathrm{MeV} / N$ calculated with the impulse approximation and the first Born approximation (B). The ratio is shown as a function of electron energy for various emission angles $\vartheta_{f}$.

the experimental spectrum one has to average over the angular resolution (being $1.7^{\circ}$ ) and the energy resolution $\Delta E_{f}$ (being $3.4 \%$ ) of the detector according to

$$
\begin{aligned}
& \left\langle\frac{\mathrm{d}^{2} \sigma}{\mathrm{d} E_{f} \mathrm{~d} \Omega_{f}}\right\rangle_{\vartheta_{j}, E_{f}} \\
& \quad=\frac{1}{\Delta E_{f}} \int_{E_{f}-\Delta E_{f} / 2}^{E_{f}+\Delta E_{f} / 2} \mathrm{~d} E_{f}\left(\frac{1}{1-\cos \left(1.7^{\circ}\right)} \int_{0}^{1.7^{\circ}} \sin \vartheta_{f} \mathrm{~d} \vartheta_{f} \frac{\mathrm{d}^{2} \sigma}{\mathrm{d} E_{f} \mathrm{~d} \Omega_{f}}\right)
\end{aligned}
$$

where it is from a numerical point of view important to average first over the angle as the integrand is finite at $\vartheta_{f}=0$ because of the phase factor $\sin \vartheta_{f}$. Due to the peak asymmetry, this shifts the maximum from $k_{f}=18.1$ down to 18.04 , and the average over energy shifts it further down to 18.0 in agreement with the experimental peak position (figure 7). Unfortunately the absolute cross section has not been measured such that we had to normalise the data to our calculation. Clearly, the large asymmetry of the peak is seen, and our curve compares well with the experimental shape. Actually this is also true for a second-order Born calculation (Breinig et al 1982), so that one cannot give preference to one theory by merely comparing the relative values.

\section{Conclusion}

We have calculated the doubly differential cross section for electron emission from light target atoms by using the semiclassical impulse approximation which is valid for heavy projectiles and can be applied to the evaluation of the electron spectrum except at $E_{f} \rightarrow 0, E_{f}$ being the electon energy in the laboratory frame. Although a peaking approximation is applied in the numerical calculations, it does not restrict the applicability to very high velocities $v \gg v_{i}$ as is the case for other higher-order theories discussed in the literature. Rather, the use of a straight-line path for the internuclear motion may invalidate the present formulae at low collision velocities.

The results of the present theory differ largely in absolute magnitude from the first-order Born approximation except at the binary encounter peak, and they differ 


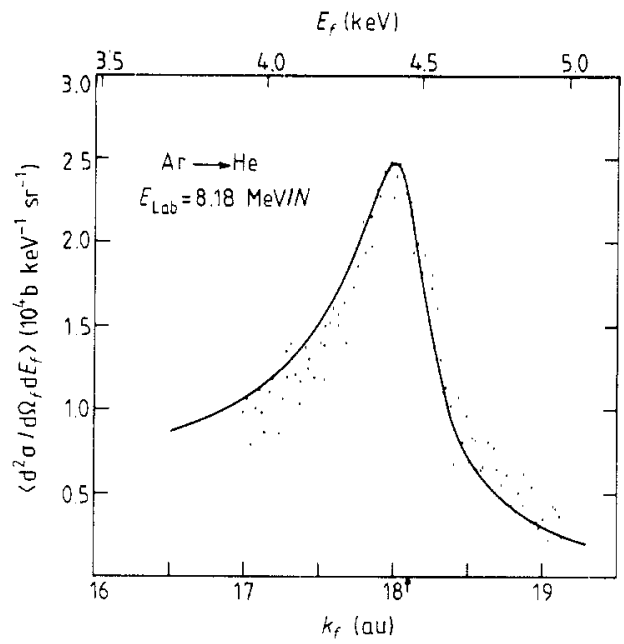

Figure 7. Averaged cross section for electron emission in collisions of Ar with $\mathrm{He}$ at a collision velocity of 18.1 au (marked with arrow) as a function of momentum (or energy) near zero emission angle. The full curve denotes SCIA calculations and the experimental data (which are normalised to theory) are from Breinig et al (1982).

also from a simple approximation to the second-order Born theory. There are very few experimental data presently available on electrons from fast and asymmetric collisions, but so far they can be explained by the scIA. More data on electron spectra, especially including absolute cross section measurements, would be welcome in order to distinguish clearly between the existing theories.

\section{Appendix}

We present in this appendix the explicit forms of the wavefunction, as well as the integral over the momentum components $s_{0 \perp}$ of the product of the Fourier transforms of wavefunction and target potential, which appears during the evaluation of the transition amplitude, in the case of a helium target.

For the He 1s wavefunction we take (Clementi and Roetti 1974)

$$
\begin{aligned}
\psi_{i}^{\mathrm{He}}(\boldsymbol{r})=\frac{1}{\sqrt{4 \pi}} & (2.5925 \exp (-1.41714 r)+1.6377 \exp (-2.37682 r) \\
& +0.75254 \exp (-4.39628 r) \\
& -0.3315 \exp (-6.52699 r)+0.103 \exp (-7.94252 r) .
\end{aligned}
$$

As the target is screened, the integral (2.8) has to be replaced by

$$
\begin{gathered}
I \equiv \int_{0}^{\infty} s_{0 \perp} \mathrm{d} s_{0 \perp} \boldsymbol{\varphi}_{i}^{\mathrm{He}}\left(\boldsymbol{s}_{0}+\boldsymbol{v}\right) \int_{0}^{2 \pi} \mathrm{d} \varphi_{s_{0}}\left(\frac{1}{\left(\boldsymbol{q}_{2}+\boldsymbol{k}_{f}-\boldsymbol{v}-\boldsymbol{s}_{0}\right)^{2}}+\frac{1}{\left(\boldsymbol{q}_{2}+\boldsymbol{k}_{f}-\boldsymbol{v}-\boldsymbol{s}_{0}\right)^{2}+d^{-2}}\right. \\
\left.+\frac{1}{d^{2}\left[\left(\boldsymbol{q}_{2}+\boldsymbol{k}_{f}-\boldsymbol{v}-\boldsymbol{s}_{0}\right)^{2}+d^{-2}\right]^{2}}\right)
\end{gathered}
$$


where the Fourier transform $\varphi_{i}^{\mathrm{He}}$ is taken from (3.4) with the parameters defined by comparison of (3.2) and (A.1). From the binding energy $E_{i}=-0.91795$ one obtains with (A.1) the parameter $d=0.3045$. Recall that all parameters are in atomic units.

In order to perform the $\varphi_{s_{0}}$ integration, we split the momentum into its components parallel to $v$ and perpendicular to it, such that

$$
\left(\boldsymbol{q}_{2}+\boldsymbol{k}_{f}-\boldsymbol{v}-\boldsymbol{s}_{0}\right)^{2}=\left(q_{2 z}+k_{f z}-v-s_{0 z}\right)^{2}+\left(\boldsymbol{q}_{2 \perp}+\boldsymbol{k}_{f \perp}-\boldsymbol{s}_{0 \perp}\right)^{2}
$$

and define $\varphi_{s_{0}}$ as the angle between $\boldsymbol{q}_{2_{-}}+\boldsymbol{k}_{f \perp}$ and $\boldsymbol{s}_{0 \perp}$. The integration is done with the help of the formulae

$$
\int_{0}^{2 \pi} \mathrm{d} \varphi \frac{1}{(A-B \cos \varphi)^{n}}=2 \pi \begin{cases}\left(A^{2}-B^{2}\right)^{-1 / 2} & n=1 \\ A\left(A^{2}-B^{2}\right)^{-3 / 2} & n=2\end{cases}
$$

The remaining integral over $s_{0 \perp}$ is straightforward. The result is

$$
\begin{aligned}
& I=2^{3 / 2} Z_{\mathrm{T}}^{5 / 2} f_{0}^{\mathrm{He}}\left(q_{2}, s_{0 z}\right) \\
f_{0}^{\mathrm{He}}\left(q_{2}, s_{0 z}\right)= & \frac{1}{2} \sum_{i=1}^{5} c_{i} \xi_{i}\left(\frac{1}{\beta_{i}}\left(\frac{q_{0}^{2}+p_{\perp}^{2}}{p_{i}^{2}}-1\right)-\frac{\alpha_{i}}{2 \beta_{i}^{3 / 2}} \ln \frac{2 \sqrt{\beta_{i}}\left(q_{0}^{2}+p_{\perp}^{2}\right)+2 \beta_{i}+\alpha_{i} p_{i}^{2}}{p_{i}^{2}\left(\alpha_{i}+2 \sqrt{\beta_{i}}\right)}\right. \\
& +\frac{1}{\beta_{i 0}}\left(\frac{p_{1}^{2}}{p_{i}^{2}}-1\right)+\frac{1}{d^{2}}\left\{-\frac{3 \alpha_{i 0}}{2 \beta_{i 0}^{2}}+\frac{2 p_{\perp}^{2}}{\beta_{i 0}^{2}\left(4 \beta_{i 0}-\alpha_{i 0}^{2}\right)}\left[\left(3 \alpha_{i 0}^{2}-8 \beta_{i 0}\right)\left(1-\frac{p_{i}^{2}}{p_{1}^{2}}\right)\right.\right. \\
& \left.\left.-\frac{\alpha_{i 0}}{p_{1}^{2}}\left(3 \alpha_{i 0}^{2}-10 \beta_{i 0}\right)\right]+\frac{1}{\beta_{i 0} p_{1}^{2}}\left(\frac{3 \alpha_{i 0} p_{i}^{2}-2 \beta_{i 0}+3 \alpha_{i 0}^{2}}{2 \beta_{i 0}}+\frac{\alpha_{i 0}+4 p_{\perp}^{2}}{2 p_{i}^{2}}\right)\right\} \\
& \left.+\frac{1}{2 \beta_{i 0}^{3 / 2}}\left(-\alpha_{i 0}+\frac{2}{d^{2}}-\frac{3 \alpha_{i 0}}{\beta_{i 0} d^{2}}\left(\frac{1}{2} \alpha_{i 0}+2 p_{\perp}^{2}\right)\right) \ln \frac{2 \sqrt{\beta_{i 0}} p_{1}^{2}+2 \beta_{i 0}+\alpha_{i 0} p_{i}^{2}}{p_{i}^{2}\left(\alpha_{i 0}+2 \sqrt{\beta_{i 0}}\right)}\right)
\end{aligned}
$$

with the abbreviations from (2.8) and

$$
\begin{array}{ll}
p_{i}^{2}=\xi_{i}^{2}+\left(s_{0 z}+v\right)^{2} & p_{1}^{2}=q_{0}^{2}+p_{\perp}^{2}+d^{-2} \\
\alpha_{i}=2\left(q_{0}^{2}-p_{\perp}^{2}-p_{i}^{2}\right) & \alpha_{i 0}=\alpha_{i}+2 d^{-2} \\
\beta_{i}=\left(q_{0}^{2}+p_{\perp}^{2}-p_{i}^{2}\right)^{2}+4 p_{\perp}^{2} p_{i}^{2} & \beta_{i 0}=\left(p_{1}^{2}-p_{i}^{2}\right)^{2}+4 p_{\perp}^{2} p_{i}^{2} .
\end{array}
$$

\section{References}

Abramowitz M and Stegun I A 1965 Handbook of Mathematical Functions (New York: Dover) p 556 Band Y B 1974 J. Phys. B: At. Mol. Phys. 72557

Breinig M, Elston S B, Huldt S, Liljeby L, Vane C R, Berry S D, Glass G A, Schauer M, Sellin I A, Alton G D, Datz S, Overbury S, Laubert R and Suter M 1982 Phys. Rev. A 253015

Briggs J S 1977 J. Phys. B: At. Mol. Phys. 103075

Clementi E and Roetti C 1974 At. Data Nucl. Data Tables 14177

Dettmann K, Harrison K G and Lucas M W 1974 J. Phys. B: At. Mol. Phys. 7269

Faddeev L D 1961 Sov. Phys.-JETP 121014

Folkmann F, Groeneveld K O, Mann R, Nolte G, Schumann S and Spohr R 1975 Z. Phys. A 275229

Garibotti C R and Miraglia J E 1980 Phys. Rev. A 21572

Jakubassa-Amundsen D H 1981 J. Phys. B: At. Mol. Phys. 143139

Jakubassa-Amundsen D H and Amundsen P A 1980 Z. Phys. A 297203

-1981 J. Phys. B: At. Mol. Phys. 14 L705

Lucas M W, Steckelmacher W, Macek J and Potter J E 1980 J. Phys. B: At. Mol. Phys. 134833

McDowell M R C 1961 Proc. R. Soc. A 264277 
Macek J 1970 Phys. Rev. A 1235

Macek J and Alston S 1982 Phys. Rev. A 26250

Miraglia J E and Ponce V H 1980 J. Phys. B: At. Mol. Phys. 131195

Nielsen S E and Dahler J S 1977 Phys. Rev. A 16563

Nordsieck A 1954 Phys. Rev. 93785

Okubo S and Feldman D 1960 Phys. Rev. 117292

Oldham W J B Jr 1967 Phys. Rev. 1611

Rudd M E, Sautter C A and Bailey C L 1966 Phys. Rev. 15120

Salin A 1969 J. Phys. B: At. Mol. Phys. 2631

Shakeshaft R and Spruch L 1978 Phys. Rev. Lett. 411037

Wadehra J M, Shakeshaft R and Macek J H 1981 J. Phys. B: At. Mol. Phys. 14 L767 